\title{
Preface
}

\section{Innovations in and Around the Foregut}

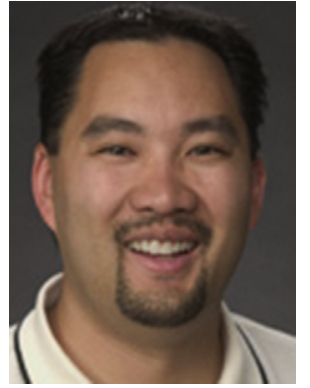

Brian E. Louie, MD, MHA, MPH, FRCSC, FACS

Editor

The esophagus has a deceptively simple role in the human body: deliver orally ingested material from the mouth to the stomach. Yet, it is involved in and the source of much disease from the highly prevalent gastroesophageal reflux (GERD) to the more rare motility disorders such as achalasia, and last, the Barrett-adenocarcinoma pathway. Over the last several years, considerable progress and changes in diagnostic tools and approaches to surgical treatments of esophageal disease have been made. In this issue of Thoracic Surgery Clinics, we examine a cadre of new innovations and some that have been around for some time.

The first content theme surrounds the management of Barrett esophagus. Diagnosis has evolved from endoscopic biopsies using the Seattle protocol with new diagnostic tools, such as wide area transepithelial sampling, optical coherence tomography, and confocal laser endomicroscopy to enhance the detection and diagnosis of Barrett esophagus along the continuum from nondysplastic to dysplasia. With early detection, subsequent treatment before cancer has developed can be initiated and involve endoscopic resections and ablative therapies.

Open operations gave way to laparoscopic approaches, and we are witnessing another paradigm shift as endoscopic surgery takes us into the potential space of the submucosa: the highway of the esophagus. Per oral endoscopic myotomy has been rapidly adopted around the world as an alternative to laparoscopic myotomy. This has also opened up additional options to address diseases related to the cricopharyngeal muscle
(Zenker diverticulum) and the pylorus (gastroparesis) with flexible endoscopy.

Nissen fundoplication and its modifications have been the only surgical option for the management of GERD for 70 years. The surgical armamentarium for GERD has more than doubled with viable options that focus on restoring the LES function and seek to balance the control of GERD with the potential side effects of Nissen. The procedures involve extraluminal devices, such as magnetic sphincter augmentation and electrical stimulation of the lower esophageal sphincter, and endoluminal approaches, like transoral incisionless fundoplication. The difficult hiatus during repair of the large hiatal hernia remains a challenge as evidenced by high recurrence rates. Surgical adjuncts, such as mesh, gastroplasty, and relaxing incisions, have been added to simple suture closure to mitigate against the tensions found at the esophageal hiatus during repair.

Even the management of perforations and anastomotic problems has moved endoluminal. Most thoracic surgeons are acquainted with the use of clips and stents, but the use of endoluminal wound vacuum therapy has emerged as a viable option to not only stents, but also diversion and exclusion.

Poised to influence the diagnosis and treatment of esophageal diseases are the functional endoluminal probes that may guide therapy in achalasia or esophageal tissue impedance as a new diagnostic test for GERD.

The problem of gastric pull-up perfusion during esophagectomy is delineated by the use of fluorescence imaging and can be used to select an 
appropriately vascularized location for the esophagogastric anastomosis in hopes of lowering the anastomotic complication rate.

Last, a careful look at how the adoption and integration of these new techniques into clinical practice in terms of training, credentialing, and evaluation should occur is examined.

I want to thank all the authors for their time and contributions to this issue of Thoracic Surgery Clinics. I hope that you'll enjoy reviewing these articles and find something that is innovative that can be useful in your thoracic surgical practice.

Brian E. Louie, MD, MHA, MPH, FRCSC, FACS Division of Thoracic Surgery Swedish Cancer Institute

Suite 900

1101 Madison Street Seattle, WA 98104, USA

E-mail address: brian.louie@swedish.org 